\title{
ERRATA, VOLUME 14
}

K. N. Srivastava, $A$ class of integral equations involving ultraspherical polynomials as kernel, pp. 932-940.

Page 934, Equation (9): “ $C_{n-1}^{\lambda}(u / v)$ " should read " $C_{n-1}^{-\lambda}(u / v)$."

Page 936, Equation (13):

$$
\text { "[ }\left[\frac{n-2}{2}\right] \text { " should read " }\left[\frac{n-1}{2}\right] \text { ". }
$$

\section{ERRATA, VOLUME 15}

Culbreth Sudler Jr., Two algebraic identities and the unboundedness of a restricted partition function, pp. 16-20.

Page 18, line 12 (second term in expression for $g_{u}(x)$ ). The exponent should read $t u+t(t+1) / 2$.

G. M. Bergman, A ring primitive on the right but not on the left, pp. $473-475$.

Page 474, line 6. Read $n>j$ for $n>0$.

Line 10. For the last $2^{n-j-1}$, read $2^{n-i}$.

Line 24. After "subring," insert "of $A$."

Shreeram Abhyankar, A remark on the nonnormal locus of an analytic space, pp. 505-508.

Line 5 on page 507 which now reads " $K$ into $L . \cdots g\left(R^{\prime}\right)=S^{\prime}$." should be changed to read " $K$ into L. Let $S^{*}$ be the quotient ring of $g\left(R^{\prime}\right)$ with respect to $g(M)$ where we regard $S^{*}$ to be a subring of $L$. Assume that $\mathrm{g}(K)=L$. Then $S^{\prime}=S^{*}$."

Lines 9 to 14 on page 507 which now read " $g\left(R^{\prime}\right)=S^{\prime}$. Now . . normal." should be changed to read " $S^{\prime}=S^{*}$. Now assume that furthermore $\mathfrak{c}(R) \cap M \neq \varnothing$. Fix $w \in \mathfrak{c}(R) \cap M$. Since $S^{\prime}=S^{*}$, given any $z \in S^{\prime}$ there exists $w^{\prime} \in R^{\prime}$ and $w^{*} \in M$ such that $z=g\left(w^{\prime}\right) / g\left(w^{*}\right)$; since $w \in M$, upon multiplying the numerator and the denominator by $g(w)$ we get that $z=g\left(w w^{\prime}\right) / g\left(w w^{*}\right)$; since $w^{\prime} \in R^{\prime}$ and $w \in \mathfrak{c}(R)$ we get that $w w^{\prime} \in R$; now $w w^{*} \in M$ and hence $z \in S$. Thus $S^{\prime}=S$, i.e., $S$ is normal."

The third and the fourth sentences in the last paragraph on page 507 which now read "Since $g(R) C S, \ldots$ be given." should be changed to read "Therefore by [5, Lemma 2 on p. 257] we get that $S^{*}$ is integral over $S$ and hence $S^{*} \subset S^{\prime}$. To show that $S^{\prime} \subset S^{*}$, let $x^{\prime} \in S^{\prime}$ be given."

The last two sentences on page 508 which now read "Since $m m^{\prime} \in M, \cdots$ hence $x^{\prime} \in g\left(R^{\prime}\right)$." should be changed to read "Now $m m^{\prime} \in M$ and $x^{\prime}=g(t) / g\left(m m^{\prime}\right)$. Therefore $x^{\prime} \in S^{*}$." 